\title{
PAPER
}

\section{An auditory model that can account for frequency selectivity and phase effects on masking}

\author{
Akira Nishimura $^{1, *}$ \\ ${ }^{1}$ Department of Media and Cultural Studies, Faculty of Informatics, Tokyo University of \\ Information Sciences, 1200-2, Yato-cho, Wakaba-ku, Chiba, 265-8501 Japan
}

(Received 26 August 2003, Accepted for publication 9 April 2004)

\begin{abstract}
A peripheral auditory model that accounts for masking by the positive and negative Schroeder-phase complex maskers was proposed. In addition, the proposed model provides reasonable frequency selectivity whereas the previous model using the analytic gammachirp filter did not. The proposed model is a cascade of a fixed filter for outer/middle ear response, a compressive gammachirp filterbank for auditory filters, a half-wave rectifier, a direct current component adder, a leaky integrator, and a detection module. Masking data of a short signal by the Schroeder-phase complex masker and notched-noise masking data were collected from the same human listeners to measure phase characteristics and frequency selectivity of the auditory periphery. The peripheral auditory model was used to simulate masking of a short signal, and the power spectrum model of masking was used to simulate the notched-noise masking data. Parameter fittings of both models were conducted simultaneously using the same parameter values of the compressive gammachirp filter for the individual and mean data. The results of model parameter fitting for both models provide a good explanation for human masking data.
\end{abstract}

Keywords: Schroeder-phase complex, Compressive gammachirp filter, Masking period pattern, Notched noise, Power spectrum model

PACS number: 43.66.Ba, 43.66.Dc, 43.66.Mk, 43.66.Nm [DOI: 10.1250/ast.25.330]

\section{INTRODUCTION}

Phase effects in masking experiments using harmonic complex maskers are usually associated with strong variations in the masker envelope. Representative examples are positive and negative Schroeder-phase complexes. A Schroeder-phase complex having a fundamental frequency of $f_{0} \mathrm{~Hz}$ is given by

$$
\begin{gathered}
S(t)=\sum_{m=n_{1}}^{n_{\mathrm{h}}} \sin \left(2 \pi m f_{0} t+\operatorname{sign} \times \operatorname{phase}(m)\right), \\
\operatorname{phase}(m)=\pi m(m-1) /\left(n_{\mathrm{h}}-n_{1}+1\right),
\end{gathered}
$$

where $n_{\mathrm{l}}$ is the lowest harmonic number and $n_{\mathrm{h}}$ is the highest harmonic number. When sign is positive, $S(t)$ represents a positive Schroeder-phase complex (POS), and when sign is negative, $S(t)$ represents a negative Schroederphase complex (NEG). POS and NEG are time-reversed and inverted versions of each other. Both phase relationships produce very flat envelopes. However, masked thresholds of a pure tone with frequency and phase

\footnotetext{
*e-mail: akira@rsch.tuis.ac.jp
}

identical to a component of the Schroeder-phase masker vary by approximately $20 \mathrm{~dB}$ between POS and NEG maskers [1,2].

Several studies [1-5] have reported that these masking differences are due to phase characteristics of the auditory filter. It has been reported that negative curvature of the auditory filter phase characteristics, that is, an upward frequency glide in the impulse response, creates pronounced peaks and dips in the POS waveform envelope. In addition, this causes the NEG waveform envelope to flatten.

Kohlrausch and Sander [2] have pointed out that the different shapes of the masking period patterns (MPPs) for POS and NEG maskers are inconsistent with the gammatone filter characteristics proposed by Patterson et al. [6]. The main reason is that both POS and NEG waveforms at the output of the gammatone filter have the same envelopes because of the curvature of their phase functions, which is antisymmetric about its center frequency. Smith et al. [1] and Kohlrausch and Sander [2] utilized the one-dimensional basilar membrane model proposed by Strube [7] as an auditory filter to account for the phase effect on 
masking. However, they did not show quantitative analysis of the threshold variations between the POS and NEG maskers.

Carlyon and Datta [4] measured MPPs with respect to the sound pressure level, the number of components, and the phase of flanking components of the Schroeder-phase maskers. They showed that several features in the data could be simulated using a nonlinear model of the auditory periphery as described by Giguére and Woodland [8]. The only modification to the model was a change in parameter $Q_{n}$ which describes the sharpness of the basilar membrane tuning from its default value of 2 to the sharper value of 8 , in order to obtain reasonable frequency selectivity. However, the forms of the simulated MPPs did not coincide with those of the experimental MPPs. In addition, it is not clear that the frequency selectivity of the model is appropriate for that of a human listener.

Oxenham and Dau [9] have investigated several types of auditory filters, including the analytic gammachirp filter, to account for both the dependence of masked thresholds on the phase of the Schroeder-phase complex and the frequency selectivity of these auditory filters. In simulations using the model of masking proposed by Dau et al. [10], none of the auditory filters could quantitatively account for both masking data and frequency selectivity.

The author [5] has proposed a peripheral auditory model that adopts the analytic gammachirp filter [11] as an auditory filterbank. This model was able to simulate the difference in masked threshold between POS and NEG observed in previous studies $[1,2,4]$. However, a large negative value is required for $c$, a parameter of the analytic gammachirp filter, in order to obtain the appropriate negative curvature of the phase function. This causes the amplitude spectrum of the filter to have a steeper slope at high frequencies and a shallower slope at low frequencies compared to the shape of the auditory filter measured by the notched-noise method.

In this study, the analytic gammachirp filter is replaced with a compressive gammachirp filter [12] as the auditory filter of the model previously proposed by the author to account for both the MPP data of the Schroeder-phase masker and the frequency selectivity of the auditory periphery.

\section{MASKING EXPERIMENTS}

The notched-noise method is often used to measure peripheral frequency selectivity, and MPPs for a POS or NEG masker are considered a representation of an asymmetric phase function of auditory filtering. The masked thresholds obtained from both masking experiments should be verified using an auditory filter that appropriately represents the mechanism of the auditory periphery. In addition, individual differences in masking data can be accounted for by the difference in peripheral characteristics of the individual auditory systems, i.e., the differences in individual parameter values of the appropriate auditory model.

To investigate these relationships, MPP data and notched-noise data were collected from the same listeners. Using these masking data for each listener, parameters of the auditory model were then determined.

\subsection{Experimental Procedures and Apparatus}

This section describes the general experimental procedures and apparatus used for the masking experiments of this study.

All stimuli were generated digitally with 16 bit resolution at a sampling rate of $24 \mathrm{kHz}$ using a Sound Blaster Gold 64 sound card. The card was installed in an ISA bus slot of a computer. The stimuli were played from the left earpiece of a Sennheiser HDA200 headset exhibiting a frequency response that varied no more than $6 \mathrm{~dB}$ from 200 to $2000 \mathrm{~Hz}$ (B\&K 4153 IEC coupler with $B \& K$ 4134). SPLs presented in this paper were measured at $1100 \mathrm{~Hz}$ only. In all masking experiments, the electrical signal at the input to the headphone was not corrected.

Four listeners, each with absolute thresholds within $15 \mathrm{~dB}$ relative to the absolute thresholds defined by the Japan Industrial Standard, listened to the stimuli in an anechoic chamber. A two-alternative forced-choice paradigm was employed, that is, each listener was asked to select the masker containing the target signal. A 70.7\% correct detection threshold was estimated using a twodown one-up adaptive procedure, with feedback provided at the end of each two interval forced-choice trial. In the experiments to obtain the MPPs, the signal level was varied. In the notched-noise experiment, the signal level was fixed and the notched masker level was varied. The step size of the level increment and decrement was $4 \mathrm{~dB}$ at the first three turnpoints and $2 \mathrm{~dB}$ after the third turnpoint. Each run terminated after 12 turnpoints, and the estimate for that run was obtained by averaging the levels of the last eight turnpoints. All data presented here were obtained from the mean of at least six runs on separate days.

\subsection{Experiment 1: Measuring the Masking Period Patterns of the Schroeder-Phase Complexes}

MPPs were measured for two maskers, POS and NEG, each consisting of equal-amplitude harmonics 2-20 of a $100-\mathrm{Hz}$ fundamental. This means that $n_{1}=2$ and $n_{\mathrm{h}}=20$ in Eqs. (1) and (2). Each MPP was measured at a masker level of $55 \mathrm{~dB} \mathrm{SPL} /$ component. In all cases the masker duration was $250 \mathrm{~ms}$, including two 20-ms raised-cosine ramps, and the signal was a $5-\mathrm{ms} 1,100-\mathrm{Hz}$ sinusoid composed of two 2.5-ms raised-cosine ramps (no steady state) added in-phase to the $1,100-\mathrm{Hz}$ masker component. 


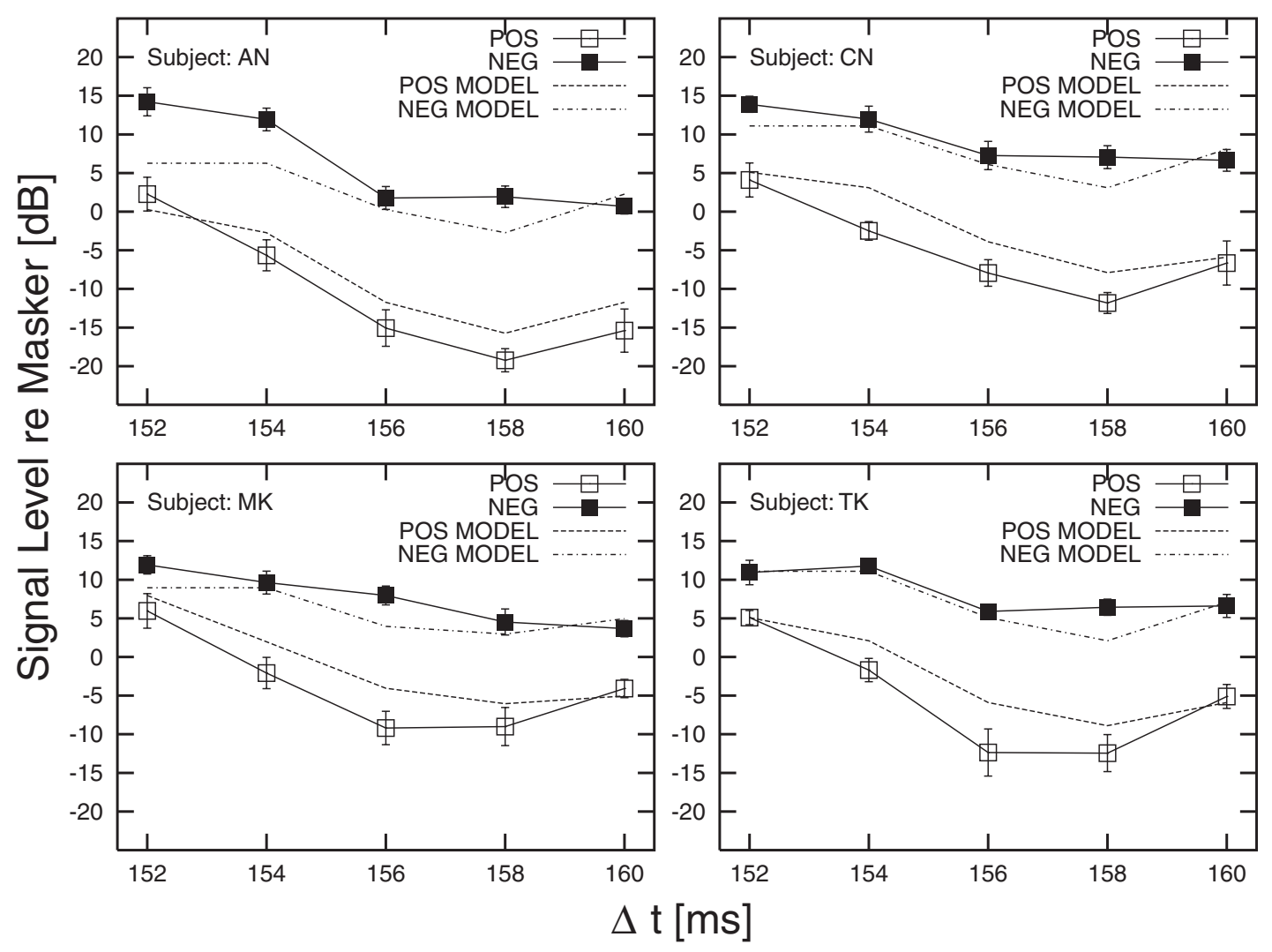

Fig. 1 Results of experiment 1 and predictions by the auditory model. Error bars denote plus and minus one standard deviation.

The detailed procedure for measuring masked thresholds is described in Sect. 2.1. The signal was turned on at a $\Delta t=152,154,156,158$ or $160 \mathrm{~ms}$ after the beginning of the masker (zero-voltage points). These conditions were identical to those of the first experiment conducted by Carlyon and Datta [4], with the exception that they used masker levels of 39 to $69 \mathrm{~dB}$ SPL/component and a masker duration of $400 \mathrm{~ms}$.

Figure 1 shows the results of Experiment 1 for each subject together with data obtained from the model predictions described in Sect. 3. The error bars represent plus and minus one standard deviation from the mean. All data points are expressed relative to the level of a single masker component. MPPs obtained from each subject show tendencies similar to those reported by Carlyon and Datta [4]. The MPPs for POS have a minimum at $\Delta t=158 \mathrm{~ms}$. The MPPs for NEG show little variation over a period compared to the POS masker.

\subsection{Experiment 2: Manipulating the Phase of Off- Frequency Components}

Experiment 2 investigated the effect of the phase of offfrequency components on the masking of POS and NEG. The masker always contained harmonics $2-20$ of a $100-\mathrm{Hz}$ fundamental, and phases of components remote from 1,100 $\mathrm{Hz}$ were manipulated. In other words, Experiment 2 examined the number of components required to produce a large difference in the masked threshold between NEG and POS maskers, especially at $\Delta t=158 \mathrm{~ms}$ as shown in Experiment 1. Thus, the results of Experiment 2 reflect frequency selectivity in the auditory periphery.

For the first condition, the central $M$ components were selected from the POS masker of Experiment 1, and the remaining components were selected from the NEG masker of Experiment 1. For example, if $M=5$, masker components between $900 \mathrm{~Hz}$ and $1,300 \mathrm{~Hz}$ are positive phase and the remaining components of $200 \mathrm{~Hz}$ to $800 \mathrm{~Hz}$ and $1,400 \mathrm{~Hz}$ to $2,000 \mathrm{~Hz}$ are negative phase. For the second condition, the central $M$ components were selected from the NEG masker and the remaining components were selected from the POS masker. In both conditions, masked thresholds were obtained for the $5-\mathrm{ms} 1,100-\mathrm{Hz}$ signal at $\Delta t=158 \mathrm{~ms}$ (the minimum of the positive-phase MPP) as a function of $M$. The detailed procedure for obtaining the masked thresholds is described in Sect. 2.1. $M$ was selected from 5, 7, 9 and 11. In POS condition, $M=0$ denotes NEG condition and $M=19$ denotes POS condition in Experiment 1 and vice versa. Therefore, data were collected from Experiment 1 under the conditions of $M=0$ and 19. Other parameters were the same as in Experiment 1. The experimental design was based on the third experiment of Carlyon and Datta [4]. 


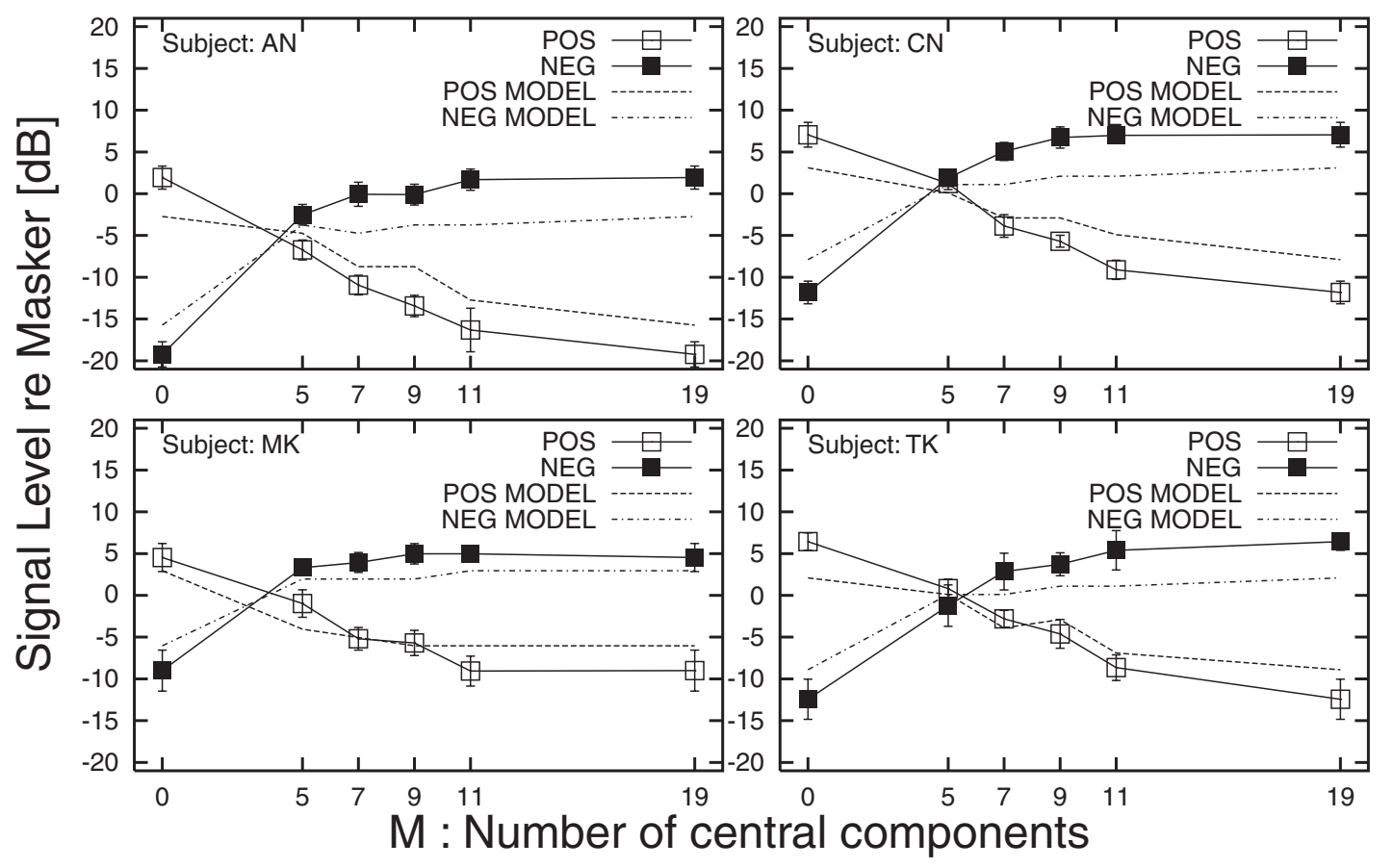

Fig. 2 Results of experiment 2 and predictions by the auditory model. Error bars denote plus and minus one standard deviation.

Results for each subject are shown in Fig. 2, along with predictions of the model simulations described in Sect. 3 . The error bars represent plus and minus one standard deviation from the mean. All data points are expressed relative to the level of a single masker component. The experimental data also show tendencies similar to those reported by Carlyon and Datta [4]. The averaged threshold for each subject is significantly higher when the nine harmonics between 700 and $1,500 \mathrm{~Hz}$ are positive in phase than when the 11 harmonics between 600 and $1,600 \mathrm{~Hz}$ are positive in phase (one-tailed $t$-test $p<0.012$ for each subject). This means that components more than $400-\mathrm{Hz}$ distant from the $1,100-\mathrm{Hz}$ signal affect its threshold.

\subsection{Experiment 3: Masking by Notched-Noise}

Masked thresholds were determined for a sinusoidal probe tone $f_{\mathrm{p}}$ of $1,100 \mathrm{~Hz}$ in the presence of notched-noise maskers having variable notch widths. The probe level was fixed to $50 \mathrm{~dB}$ SPL, and the noise level was varied to determine thresholds. The detailed procedure for obtaining the masked thresholds is described in Sect. 2.1. A preliminary experiment showed the spectral level of the masker of a flat (no-notch) spectrum at the masked threshold of the target was approximately $55 \mathrm{~dB}$ SPL/ $100 \mathrm{~Hz}$, equal to the masker level of the MPP experiments described in the previous sections. Consequently, the probe level of $50 \mathrm{~dB}$ SPL may produce the same excitation level at the output of the auditory filter around $1,100 \mathrm{~Hz}$ for both notched-noise masking and the MPP experiments.
The notches were placed both symmetrically and asymmetrically about the probe frequency. The outside edges of the masker noise were fixed at $0.2 f_{\mathrm{p}}$ and $1.8 f_{\mathrm{p}}(220$ and $1,980 \mathrm{~Hz}$ ). The frequencies of the notch edges were specified in normalized frequency units relative to the probe frequency as given by $\left(\left|f-f_{\mathrm{p}}\right|\right) / f_{\mathrm{p}}$. In the symmetric conditions, both notch edges were placed at the normalized values of $0,0.1,0.2,0.3$ and 0.4 . In the asymmetric conditions, one of the notch edges was set at a normalized value of $0,0.1,0.2$ and 0.3 , whereas the other was set 0.2 normalized unit further away, that is, at the normalized value of $0.2,0.3,0.4$ and 0.5 times away from $f_{\mathrm{p}}$.

The duration of the probe signal was $400 \mathrm{~ms}$, including 10 -ms raised-cosine ramps. The probe was temporally centered within the masker, which consisted of a 500-ms duration including 10-ms raised-cosine ramps. To generate the masker, a random Gaussian noise was Fourier transformed and filtered in the frequency-domain. A 65,536points inverse FFT was then applied to generate the time waveform. At the start of each threshold determination, a 2.73-second buffer of the notched noise was generated for use during this test. In each trial, a 500-ms portion of the buffer was chosen randomly for each of the two masker intervals within each trial. Results are shown in Fig. 3 for each subject, along with the data predicted by the power spectrum model of masking described in the next section. The error bars represent plus and minus one standard deviation from the mean. 


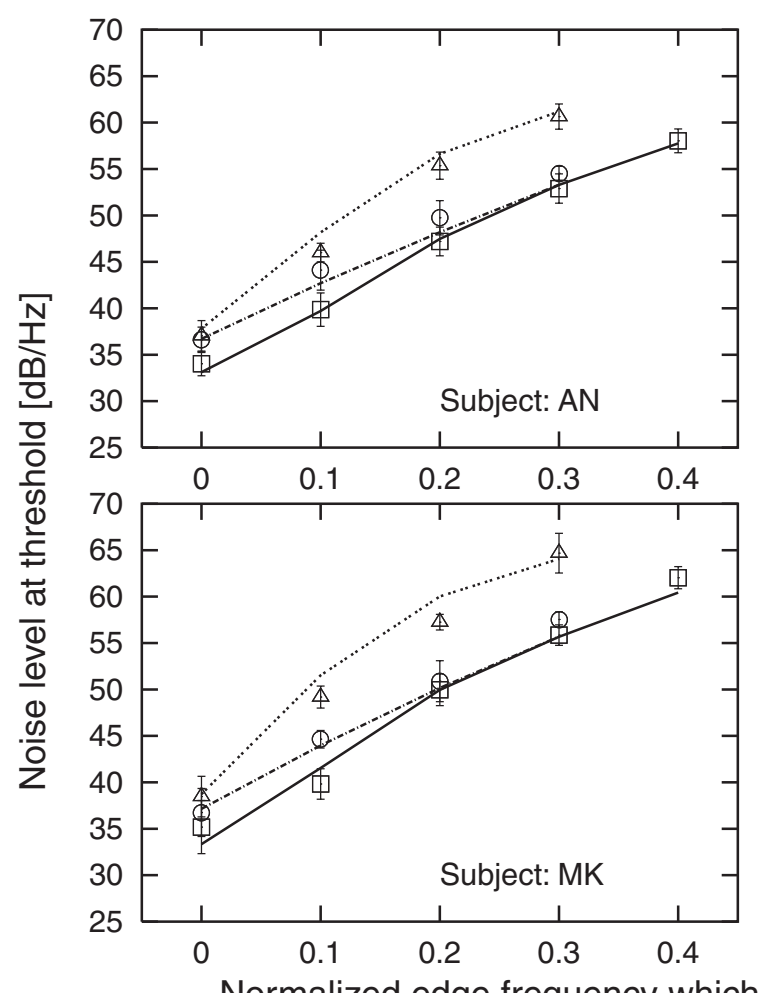

Normalized edge frequency which is MODEL lower asymmetric notch - - - MODEL higher asymmetric notch - - MODEL symmetric
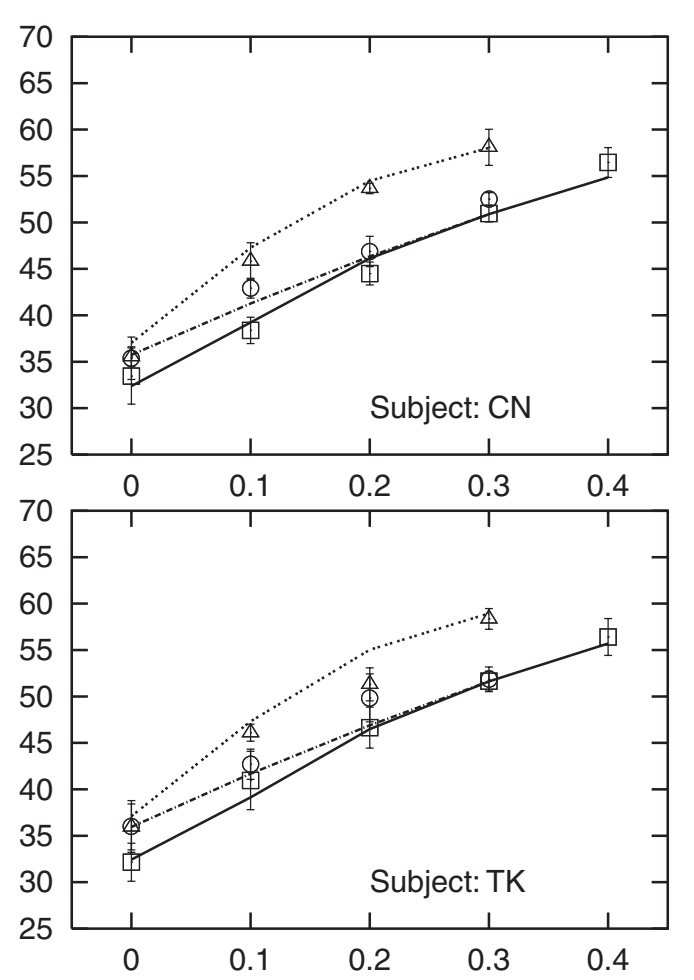

lower asymmetric notch $\triangle$ higher asymmetric notch $\bigcirc$ symmetric

Fig. 3 Results of the notched-noise experiment and predictions by the power spectrum model of masking. Error bars denote plus and minus one standard deviation.

\begin{tabular}{|c|c|c|c|c|c|c|}
\hline & & \multirow[b]{2}{*}{$\rightarrow \begin{array}{c}\text { Basilar } \\
\text { membrane }\end{array}$} & \multirow[b]{2}{*}{$\begin{array}{c}\text { Inner } \\
\text { hair cell }\end{array}$} & \multirow[b]{2}{*}{$\begin{array}{l}\text { (Absolute } \\
\text { threshold) }\end{array}$} & \multirow{2}{*}{\multicolumn{2}{|c|}{ Limitation of neural $\stackrel{\text { Excitation }}{\text { pattern }}$}} \\
\hline & & & & & & Detector \\
\hline & Stimulus $\rightarrow \frac{\begin{array}{c}\text { outer/middle } \\
\text { ear }\end{array}}{\begin{array}{c}\text { ELC } \\
\text { correction }\end{array}}$ & $\begin{array}{l}\text { Compressive } \\
\text { gammachirp } \\
\text { filterbank }\end{array}$ & $\begin{array}{c}\text { Half-wave } \\
\text { rectifier }\end{array}$ & $\begin{array}{l}\text { Add DC } \\
\text { component }\end{array}$ & Leaky integrator & MAX( $\left.\frac{\text { Signal+Masker }}{\text { Masker }}\right)$ \\
\hline
\end{tabular}

Fig. 4 Diagram of the auditory model.

\section{MODEL SIMULATION}

The peripheral auditory model previously proposed by the author [5] was revised to provide better agreement with the MPP data of the Schroeder-phase masker as well as reasonable frequency selectivity. The power spectrum model of masking was used to account for frequency selectivity. Both models adopt the compressive gammachirp filter with the same parameter values for the peripheral auditory filter. Therefore, the notched noise data were used as a constraint for the frequency selectivity of the auditory model. This section presents details of the models and shows how to fit the model parameters to account for the masking data.

\subsection{Overview of the Model}

The proposed auditory model consists of a series of submodules that simulate specific functions of the auditory periphery. Figure 4 shows a schematic diagram of the auditory model. The submodules are: a fixed filter for outer/middle ear response (ELC correction [13]); a compressive gammachirp filterbank for auditory filters [12]; a half-wave rectifier; a direct current (DC) component adder; and a leaky integrator. A detector is connected to the output of the leaky integrator.

A compressive gammachirp filter is produced by cascading an analytic gammachirp filter and a high-pass asymmetric function. The amplitude spectrum of the compressive gammachirp filter $\left|G_{\mathrm{CC}}(f)\right|$ is represented by a high-pass function (with center frequency $f_{\mathrm{r} 2}$ ) given by 


$$
\begin{aligned}
\left|G_{\mathrm{CC}}(f)\right| & =\left|G_{\mathrm{CA}}(f)\right| \exp \left(c_{2} \theta_{2}(f)\right), \\
\theta_{2}(f) & =\arctan \left(\frac{f-f_{\mathrm{r} 2}}{b_{2} \operatorname{ERB}\left(f_{\mathrm{r} 2}\right)}\right),
\end{aligned}
$$

where $\left|G_{\mathrm{CA}}(f)\right|$ is the amplitude spectrum of the analytic gammachirp filter and is described by the following equations. Parameters of the high-pass function, $b_{2}$ and $c_{2}$, control its transition bandwidth and slant. Then,

$$
\begin{aligned}
\left|G_{\mathrm{CA}}(f)\right| & =\left|G_{\mathrm{T}}(f)\right| \exp \left(c_{1} \theta_{1}(f)\right), \\
\left|G_{\mathrm{T}}(f)\right| & =\frac{a_{\Gamma}}{\left|2 \pi \sqrt{\left(b_{1} \operatorname{ERB}\left(f_{\mathrm{r} 1}\right)\right)^{2}+\left(f-f_{\mathrm{r} 1}\right)^{2}}\right|^{n}}, \\
\theta_{1}(f) & =\arctan \left(\frac{f-f_{\mathrm{r} 1}}{b_{1} \operatorname{ERB}\left(f_{\mathrm{r} 1}\right)}\right),
\end{aligned}
$$

where $\left|G_{\mathrm{T}}(f)\right|$ is the amplitude spectrum of the gammatone filter; $a_{\Gamma}$ is the amplitude; $n$ and $b_{1}$ are parameters defining the envelope of the gamma distribution; $c_{1}$ is the chirp factor; $f_{\mathrm{r} 1}$ is the asymptotic frequency; and $\operatorname{ERB}\left(f_{\mathrm{r} 1}\right)$ is the equivalent rectangular bandwidth whose center frequency is $f_{\mathrm{r} 1}$ [13]. When $a_{\Gamma}$ is normalized to equalize the peak amplitude of the filterbank and $n$ is fixed to 4 , the analytic gammachirp filter of the peak frequency of $f_{\mathrm{p} 1}$ is defined by two parameters, $b_{1}$ and $c_{1}$. The peak frequency of the analytic gammachirp filter $f_{\mathrm{p} 1}$ is given by

$$
f_{\mathrm{p} 1}=f_{\mathrm{r} 1}+c_{1} b_{1} \operatorname{ERB}\left(f_{\mathrm{r} 1}\right) / n .
$$

The frequency ratio between $f_{\mathrm{p} 1}$ and $f_{\mathrm{r} 2}$ is $f_{\mathrm{rat}}=f_{\mathrm{r} 2} / f_{\mathrm{p} 1}$. The impulse response of the analytic gammachirp filter is

$$
\begin{aligned}
g(t)= & a t^{n-1} \exp \left(-2 \pi b_{1} \operatorname{ERB}\left(f_{\mathrm{r} 1}\right) t\right) \\
& \times \cos \left(2 \pi f_{\mathrm{r} 1} t+c_{1} \log t\right),
\end{aligned}
$$

where $a$ is the amplitude factor and normalized to equalize the peak amplitude of the filterbank.

The compressive gammachirp filter in the proposed auditory model is defined by five parameters, $b_{1}$ and $c_{1}$ in the analytic gammachirp section, and $b_{2}, c_{2}$ and $f_{\text {rat }}$ in the high-pass asymmetric function. Consequently, frequencydependent parameters and level-dependent parameters were not adopted. The compressive gammachirp filter in this study was implemented by an IIR filter described by Irino and Unoki [14].

The main feature of the model is the phase response of the compressive gammachirp filters, emphasizing peaks in the POS masker waveform and flattening the envelope of the NEG masker waveform. The half-wave rectifier represents a simple approximation of the sum of responses of the inner hair cells. The DC component adder adds effective amplitude at the absolute threshold of a pure tone corresponding to the center frequency of the auditory filter. The leaky integrator, having the time constant of $T \mathrm{c} \mathrm{ms}$, simulates the limitation of neural synchronization of the auditory neurons. At the detection stage, absolute dif- ferences between the running output levels of the temporal integrator for each channel of masker+signal waveforms, and of the masker only (SMMR: Signal+Masker to Masker Ratio), are calculated. The maximum SMMR (MAXSMMR) is regarded as an index of detection of a short signal in the measurement of MPPs.

The signal is considered to have been detected in the frequency channel exhibiting the largest MAXSMMR, simulating off-frequency listening. The center frequencies of the auditory filters, represented by the peak frequency of the compressive gammachirp filter, were chosen in the range of $0.7 f_{\mathrm{p}} \mathrm{Hz}$ and $1.3 f_{\mathrm{p}} \mathrm{Hz}$ in steps of $0.1 \mathrm{ERB}$.

Predicted masked thresholds were obtained using the model as follows. Once the five parameter values for the compressive gammachirp, $T \mathrm{c}$ and MAXSMMR were determined, the model simulation was started with a sufficiently small signal level. The signal level was increased in 1-dB steps until the model output (SMMR) exceeded the MAXSMMR value, then the predicted signal level at the masked threshold was determined.

The most remarkable feature of the proposed auditory model for the MPP when compared to the Carlyon and Datta model is that frequency selectivity of the proposed model requires only five parameters that can also be estimated through psychoacoustic experiments, such as notched-noise masking experiments.

\subsection{Power Spectrum Model}

The shape of an auditory filter is generally derived using the power spectrum model of masking. The power spectrum model assumes that the masked threshold corresponds to a constant signal-to-masker ratio at the output of the auditory filter [13]. The compressive gammachirp filter with the five parameters described above was also adopted as an auditory filter in the power spectrum model of masking.

Once the compressive gammachirp filter is designed using the five parameters and a constant signal-to-masker ratio (signal detector efficiency $K$ ) is determined by averaging signal-to-masker ratios obtained from different notch conditions, the power spectrum model can then predict the spectral level of the notched-noise that masks a constant probe signal in different notch conditions. In the model simulation, the center frequencies of the auditory filters, represented by the peak frequency of the compressive gammachirp filter, were chosen in the range of $0.7 f_{\mathrm{p}}$ $\mathrm{Hz}$ and $1.3 f_{\mathrm{p}} \mathrm{Hz}$ in steps of $0.1 \mathrm{ERB}$. The signal was regarded as having been detected in the channel exhibiting maximum $K$.

\subsection{Fitting the Model Parameters to the Masking Data}

The simplex method by Nelder and Mead [15] was 
used to minimize an overall root mean square (RMS) difference calculated from 31 experimental data and model predictions, that is 1) the differences between the 18 masking data obtained in Experiments 1 and 2 and the predictions of the auditory model, and 2) the differences between the 13 masked thresholds obtained in Experiment 3 and the predictions of the power spectrum model of masking. All predicted values and experimental data were represented in $\mathrm{dB}$.

Parameters for fitting were $b_{1}, c_{1}, b_{2}, c_{2}$ and $f_{\text {rat }}$ for the compressive gammachirp, $T \mathrm{c}$ and MAXSMMR for the auditory model, and $K$ for the power spectrum model. Parameter values of the compressive gammachirp filter were identically controlled between the two models during the fitting procedure. Minimization of RMS difference started with the initial set of parameter values, which were reasonably defined, and iterative simulations of the two models were performed by changing the values of the parameter set according to the simplex method. Minimizing stopped when all vertices of the current simplex were within 0.05 of the best vertex [15]. Fitting procedures were conducted for each subject and for the mean data of all four subjects. Parameter fittings using the analytic gammachirp filter for the auditory model and the power spectrum model of masking were also conducted to compare the quality of fit between the proposed model and previous model [5]. In this case, the gammachirp filter was implemented using an impulse response expressed by Eq. (9) instead of an IIR implementation of the gammachirp proposed by Irino and Unoki [14], since errors in frequency and phase response of the IIR implementation increase rapidly in the range of $c<-4$.

Absolute threshold values required for the DC component in model calculation were obtained by applying cubic spline interpolation from individual absolute thresholds measured with the up-down method in 5-dB steps. A weighted mean of the two most frequent minimum audible levels in ten up-and-down sequences was adopted as the absolute threshold level. Measurement frequencies were $198 \mathrm{~Hz}$ to $2 \mathrm{kHz}$ in $1 / 3$-octave steps.

It is known that the amplitude response of the headphone affects estimation of the auditory filter shape [13]. Also, the phase response of the headphone may affect the phase effect on masking. However, it is impossible to measure both amplitude response and frequency response of a listener's outer and middle ear. And, measuring these responses in a headphone while the headphone is being worn by the listener is somewhat difficult. To simulate headphone characteristics, the impulse response of the headphone, measured by generating a time-stretched pulse signal in the IEC coupler (B\&K 4153, B\&K 4134), was convolved with the input signal of the model. The convolution was applied instead of the ELC correction of the simulation of Experiments 1 and 2. For the simulation of Experiment 3, the spectrum of the notched noise was modified according to the amplitude response of the headphone.

Table 1 shows parameter values of the model after fitting. RMS errors are shown for each experiment and the overall experiments. The data were fitted more successfully with the compressive gammachirp than with the analytic gammachirp, especially in the results of the power spectrum model of masking in Experiment 3, although several slight improvements were observed for the analytic gammachirp in the results of Experiments 1 and 2. Therefore, the compressive gammachirp filter is a reasonable choice to simultaneously account for both phase dependent masking and peripheral frequency selectivity. Figures 1, 2 and 3 show the masking data and model predictions using the compressive gammachirp filter with each subject. Although the predicted differences between masked thresholds obtained from POS and NEG are smaller than the

Table 1 Parameter values and RMS error values for fittings of the individual masking data and of the mean data for all four subjects.

\begin{tabular}{|c|c|c|c|c|c|c|c|c|c|c|c|c|}
\hline \multirow{2}{*}{ Subject } & \multicolumn{5}{|c|}{ Gammachirp filter } & \multirow{2}{*}{$\frac{\text { Efficiency }}{K[\mathrm{~dB}]}$} & \multirow{2}{*}{$\frac{\text { Leaky integrator }}{T \mathrm{c}[\mathrm{ms}]}$} & \multirow{2}{*}{$\frac{\text { Detection }}{\text { MAXSMMR }[\mathrm{dB}]}$} & \multicolumn{4}{|c|}{ RMS error $[\mathrm{dB}]$} \\
\hline & $b_{1}$ & $c_{1}$ & $b_{2}$ & $c_{2}$ & $f_{\text {rat }}$ & & & & Exp.1 & Exp.2 & Exp.3 & overall \\
\hline \multicolumn{13}{|c|}{ Fitting using the compressive gammachirp filter } \\
\hline $\mathrm{AN}$ & 1.07 & -3.71 & 5.11 & 1.59 & 0.894 & -6.38 & 1.22 & 7.61 & 4.14 & 3.70 & 1.05 & 3.09 \\
\hline $\mathrm{CN}$ & 0.936 & -3.83 & 2.20 & 1.32 & 1.53 & -5.25 & 1.25 & 10.3 & 3.04 & 3.34 & 1.17 & 2.53 \\
\hline MK & 0.912 & -3.59 & 2.68 & 0.878 & 1.04 & -6.26 & 1.29 & 9.07 & 2.93 & 2.17 & 1.44 & 2.20 \\
\hline TK & 1.01 & -3.75 & 4.66 & 1.74 & 1.21 & -5.75 & 1.22 & 10.4 & 2.99 & 2.30 & 1.54 & 2.29 \\
\hline mean & 1.14 & -3.74 & 3.57 & 1.60 & 0.978 & -6.05 & 1.20 & 11.5 & 2.62 & 2.76 & 0.85 & 2.12 \\
\hline \multicolumn{13}{|c|}{ Fitting using the analytic gammachirp filter } \\
\hline $\mathrm{AN}$ & 0.718 & -5.98 & - & - & - & -11.0 & 1.48 & 3.61 & 6.52 & 4.21 & 2.89 & 4.67 \\
\hline $\mathrm{CN}$ & 0.861 & -4.29 & - & - & - & -8.22 & 1.74 & 6.72 & 3.77 & 3.32 & 2.14 & 3.06 \\
\hline MK & 0.824 & -3.29 & - & - & - & -7.95 & 1.20 & 8.74 & 2.90 & 1.97 & 2.21 & 2.40 \\
\hline TK & 1.17 & -2.61 & - & - & - & -8.71 & 1.18 & 10.6 & 3.15 & 2.96 & 2.07 & 2.70 \\
\hline mean & 1.04 & -2.92 & - & - & - & -8.56 & 1.10 & 10.5 & 2.77 & 2.41 & 1.87 & 2.33 \\
\hline
\end{tabular}


experimental data in Figs. 1 and 2, the model predictions can simulate outlines of the MPP data for individual subjects. The predictions of the power spectrum model in Fig. 3 can also successfully simulate noise levels at the masked threshold.

These results suggest that individual differences in masking data may be accounted for in part by differences in peripheral characteristics of the individual auditory systems and differences in detection sensitivity. It seems that relatively low masked thresholds obtained from subject AN, as shown in Figs. 1 and 2, are due mainly to the low MAXSMMR at the detection threshold. Moreover, subject AN exhibits the lowest signal detector efficiency $K$ of the power spectrum model of masking. However, although large individual differences are observed in parameter values of the compressive gammachirp filter, (for example, $b_{2}$ and $f_{\text {rat }}$ of subjects $\mathrm{AN}$ and $\mathrm{CN}$ ), it does not lead to large differences in the auditory filter shapes; the filter gain differences between $\mathrm{AN}$ and $\mathrm{CN}$ are within $6 \mathrm{~dB}$ in the passband region above $-30 \mathrm{~dB}$. As for the absolute value of $c_{1}$ being larger than that of $c_{2}$, the contribution of the high-pass asymmetric function parameters $\left(b_{2}, c_{2}\right.$ and $\left.f_{\text {rat }}\right)$ to the compressive gammachirp filter amplitude response are smaller than the contribution of $b_{1}$ and $c_{1}$.

Figure 5 shows mean data for the four subjects together with model predictions after fitting. Error bars denote the maximum and minimum data of the four subjects. In this case, the overall RMS error is smallest among all fittings.

\section{DISCUSSION}

\subsection{Accounting for the Frequency Selectivity and Phase Characteristics of the Auditory Periphery}

Irino and Patterson [12] showed that the compressive gammachirp provides a reasonable fit to the level-dependent impulse response of auditory-nerve fibers in cats [16], including instantaneous frequency trajectories (that is, phase characteristics of filtering in the basilar membrane). However, a direct measurement of phase characteristics of the auditory periphery is not possible in humans. Therefore, parameter estimation of the compressive gammachirp filter for human listeners has been conducted using the power spectrum model of masking with notched-noise data [12,17].

The dependence of the phase of complex maskers on a short signal indirectly provides useful insight into phase characteristics of the auditory filter. For example, the results of Experiment 2 show that the phases of components more than $400-\mathrm{Hz}$ distant from the $1,100-\mathrm{Hz}$ signal significantly affect its threshold. The contribution to masking of these off-frequency components is known to be considerably small, according to the power spectrum model of masking, since the auditory filter attenuates these
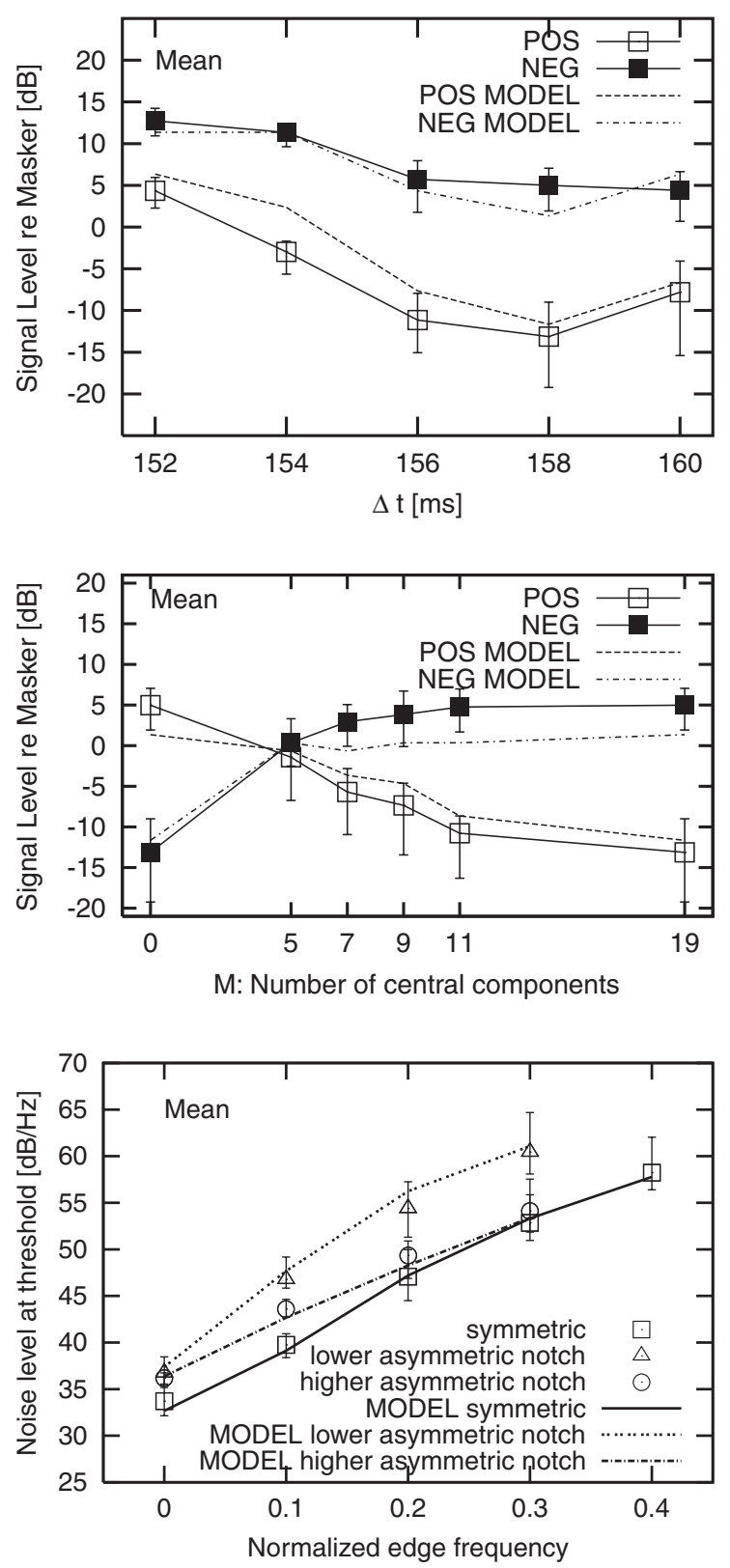

Fig. 5 Mean data for the four subjects and predictions of the model. Lines and points are the same as Figs. 1, 2 and 3 , except for error bars representing the maximum and minimum across the four subjects.

components by more than $20 \mathrm{~dB}$. Therefore, the results of Experiment 2 cannot be accounted for by the traditional power spectrum model of masking.

The previous study by the author [5] estimated parameter values of the analytic gammachirp filter using phase-dependent masking data $[1,2,4]$. However, since there was no constraint on frequency selectivity, the amplitude response of the estimated gammachirp filter was quite asymmetric compared to the auditory filter shape obtained with the notched-noise method [18].

Masking data of the short signal by the Schroederphase complex masker and notched-noise data restrict both phase and amplitude characteristics of the compressive 


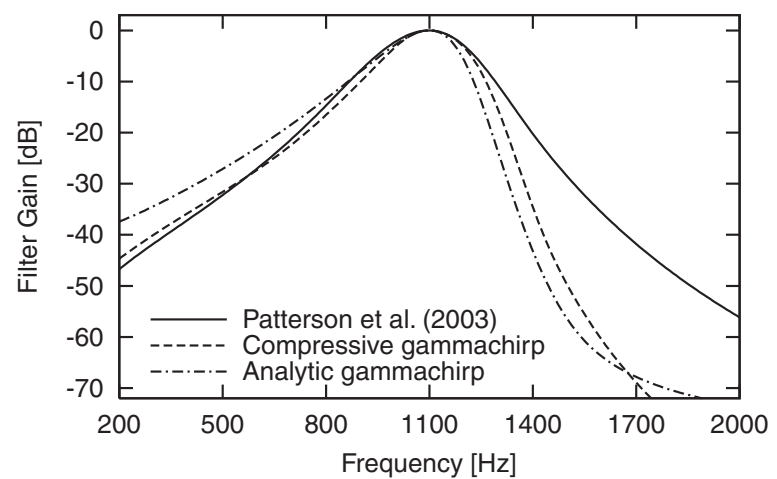

Fig. 6 Auditory filter shapes.

gammachirp filter in the fitting procedure. In the present study, the model with fitted parameter values successfully predicts masked thresholds of both types of masking experiments for individual listeners as well as for mean data for all listeners. Therefore, the amplitude and phase characteristics of the auditory periphery appear to have been realized with the compressive gammachirp filter using the parameter values for fitting determined in this study. These results provide a more realistic and meaningful auditory filter for predicting auditory masking phenomena.

\subsection{Comparison of Peripheral Parameters between Previous Studies and Current Study}

Figure 6 compares the amplitude spectra of the three auditory filters centered at $1,100 \mathrm{~Hz}$ as estimated by the data of psychoacoustic experiments. The solid line shows the results of Patterson et al. [17] for which the compressive gammachirp filter was fitted to large notched-noise data sets to define the filter parameters over center frequencies from 0.25 to $6.0 \mathrm{kHz}$. The ERB of their sixparameter compressive gammachirp filter at $P_{\mathrm{pgc}}=58 \mathrm{~dB}$ is $241 \mathrm{~Hz}$. This is approximately 1.7 times wider than the ERB at moderate stimulus levels derived from the equation suggested by Glasberg and Moore [13]. The broken line shows the compressive gammachirp filter using the parameter values obtained from the present fitting to the mean data. Its ERB is $209 \mathrm{~Hz}$. The shapes of the two filters resemble each other in the lower range. However, in the higher range, the shape of the latter filter is steeper than the former. The dash-dotted line shows the analytic gammachirp filter with parameter values obtained from the present fitting to mean data. Its ERB is $202 \mathrm{~Hz}$. The fitted analytic gammachirp filter has a steep slope in the higher range and a shallow slope in the lower range, as pointed out in the previous study [5].

The data of Experiment 3 showed that the level of the lower asymmetric notched masker required to mask the constant probe signal is greater than that of the higher asymmetric notched masker. This means that the upper side of the auditory filter is steeper than the lower side.
Predictions of the power spectrum model in Fig. 3 and the bottom panel of Fig. 5 are slightly overestimated for the lower asymmetric masker and underestimated for the higher asymmetric masker. Consequently, the upper side of the filter with the fitting of this study is estimated to be slightly steeper.

The ability of temporal resolution in human hearing has been estimated by the temporal modulation transfer function (TMTF), where the listener must detect an amplitude modulated (AM) noise signal from a nonmodulated noise signal [19]. Viemeister [19] proposed a three-stage model of modulation detection consisting of bandpass filtering followed by a half-wave rectification followed by lowpass filtering. The suggested time constant of the lowpass filter is $2.5 \mathrm{~ms}$ according to the cutoff frequency of the TMTF, whereas the time constant of the proposed model is roughly $1.2 \mathrm{~ms}$.

The discrepancy may be due to a difference in the stimuli. The waveform of the AM noise generally used to obtain the TMTF is fluctuated stochastically. Therefore, the detection process following the lowpass filter requires longer temporal integration than the lowpass filter itself. This longer temporal integration mechanism improves the low frequency region of the TMTF and lowers the cutoff frequency of the TMTF. It apparently extends the time constant of the lowpass filter. The fact that the TMTF for a 250-ms gated stimulus has a higher cutoff frequency than that of continuous or longer gated stimulus conditions (Fig. 6 in [19]) supports the above discussion.

The leaky integrator in the auditory model simulates the limitation of neural synchronization of the auditory neurons. Rhode and Greenberg [20] investigated neural temporal mechanisms in the auditory nerve and cochlea nuclei of a cat associated with the neural coding of AM tones. In their study, the temporal capability of auditory neurons was measured by computing the synchronization coefficient (SC) of the neural response to the signal's modulation and carrier frequency. The TMTF of a neuron was then computed by measuring the $\mathrm{SC}$ of the response to signals of various modulation frequencies. Although there was a significant degree of diversity in amplitude modulation coding capability among neurons in both the cochlea nuclei and auditory nerve, when the cutoff frequencies at which the SC of the TMTF falls below 0.1 were averaged over the neurons of the same response type, they were above $600 \mathrm{~Hz}$ in all unit types. In the proposed model, the TMTF of the SC for AM tones is calculated for the output waveform of the leaky integrator using a time constant of $1.2 \mathrm{~ms}$. The cutoff frequency of the TMTF is $606 \mathrm{~Hz}$. Considering the diversity of the neural response and their interaction in the cochlea nuclei, it is difficult to discuss the correspondence between the neural activities and the model. However, the time constant of $1.2 \mathrm{~ms}$ may be 
reasonable to simulate the limitation of neural synchronization.

\subsection{Type of Signal}

The present masking experiments of the Schroederphase complex dealt only with a 5-ms target signal. If a continuous signal is provided as a target, the index of signal detection MAXSMMR should be modified to another index, for example a mean value of SMMR (MEANSMMR in [5]) or a product of another temporal integration process [21].

\section{SUMMARY}

A peripheral auditory model that accounts for masking by the positive and negative Schroeder-phase complex masker was proposed. In addition, the proposed model provides reasonable frequency selectivity whereas the previous model using the analytic gammachirp filter did not. The proposed model is a cascade of a fixed filter for outer/middle ear response, a compressive gammachirp filterbank for auditory filters, a half-wave rectifier, a direct current component adder, a leaky integrator, and a detection module. At the detection stage, maximum absolute differences between the running output levels of the integrator for each channel of masker+signal waveforms, and of the masker only, is regarded as an index of detection of a short signal.

Masking data of a short signal by the Schroeder-phase complex masker and notched-noise masking data were collected from the same listeners to measure phase characteristics and frequency selectivity of the auditory periphery. The auditory model was used to simulate masking of a short signal, and the power spectrum model of masking was used to simulate the notched-noise masking data. Parameter fittings of both models were conducted simultaneously using the same parameter values of the compressive gammachirp filter for the individual and mean data. The results of model parameter fitting for both models provide good explanations for human masking data. Data were better fitted with the compressive gammachirp filter than with the analytic gammachirp filter, especially for the results of the notched-noise masking.

\section{REFERENCES}

[1] B. K. Smith, U. K. Sieben, A. Kohlrausch and M. R. Schroeder, "Phase effects in masking related to dispersion in the inner ear," J. Acoust. Soc. Am., 80, 1631-1637 (1986).

[2] A. Kohlrausch and A. Sander, "Phase effects in masking related to dispersion in the inner ear. II. Masking period patterns of short targets," J. Acoust. Soc. Am., 97, 1817-1829 (1995).
[3] R. P. Carlyon and A. J. Datta, "Excitation produced by Schroeder-phase complexes: Evidence for fast-acting compression in the auditory system," J. Acoust. Soc. Am., 101, 3636-3647 (1997).

[4] R. P. Carlyon and A. J. Datta, "Masking period patterns of Schroeder-phase complexes: Effects of level, number of components, and phase of flanking components," J. Acoust. Soc. Am., 101, 3648-3657 (1997).

[5] A. Nishimura, "An auditory model that explains masking by Schroeder-phase complexes," Acoust. Sci. \& Tech., 22, 439441 (2001).

[6] R. D. Patterson, I. Nimmo-Smith, J. Holdsworth and P. Rice, "Spiral vos final report, Part A: The auditory filterbank," Applied Psychology Unit Report, 2341 (1988).

[7] H. W. Strube, "A computationally efficient basilar-membrane model," Acustica, 58, 205-214 (1985).

[8] C. Giguére and P. C. Woodland, "A computational model of the auditory periphery for speech and hearing research. I. Ascending path," J. Acoust. Soc. Am., 95, 331-342 (1994).

[9] A. J. Oxenham and T. Dau, "Reconciling frequency selectivity and phase effects in masking," J. Acoust. Soc. Am., 110, 15251538 (2001).

[10] T. Dau, B. Kollmeier and A. Kohlrausch, "Modeling auditory processing of amplitude modulation. I. Detection and masking with narrowband carriers," J. Acoust. Soc. Am., 102, 28922905 (1997).

[11] T. Irino and R. D. Patterson, "A time-domain, level-dependent auditory filter: The gammachirp," J. Acoust. Soc. Am., 101, 412-419 (1997).

[12] T. Irino and R. D. Patterson, "A compressive gammachirp auditory filter for both physiological and psychophysical data," J. Acoust. Soc. Am., 109, 2008-2022 (2001).

[13] B. R. Glasberg and B. C. J. Moore, "Derivation of auditory filter shapes from notched-noise data," Hear. Res., 47, 103138 (1990).

[14] T. Irino and M. Unoki, "An analysis/synthesis auditory filterbank based on an IIR implementation of the gammachirp," J. Acoust. Soc. Jpn. (E), 20, 379-406 (1999).

[15] J. A. Nelder and R Mead, "A simplex method for function minimization," Comput. J., 7, 308-313 (1965).

[16] L. H. Carney, M. J. McDuffy and I. Shekhter, "Frequency glides in the impulse response of auditory-nerve fibers," J. Acoust. Soc. Am., 105, 2384-2391 (1999).

[17] R. D. Patterson, M. Unoki and T. Irino, "Extending the domain of center frequencies for the compressive gammachirp auditory filter," J. Acoust. Soc. Am., 114, 1529-1542 (2003).

[18] A. Nishimura, "Fitting a masking model to masking data with individual differences obtained from the Schroeder-phase complex masker," Trans. Tech. Comm. Psychol. Physiol. Acoust., H-2001-6, pp. 37-44 (2001).

[19] N. F. Viemeister, "Temporal modulation transfer functions based upon modulation thresholds," J. Acoust. Soc. Am., 66, 1364-1380 (1979).

[20] W. S. Rhode and S. Greenberg, "Encoding of amplitude modulation in the cochlear nucleus of the cat," J. Neurophysiol, 71, 1797-1825 (1994).

[21] A. Nishimura, "Study on the temporal integration process of the auditory model that explains masking by the harmonic complexes," Trans. Tech. Comm. Psychol. Physiol. Acoust., H-2002-7, pp. 49-56 (2002). 